\title{
A fuzzy logic-based damage identification method for simply-supported bridge using modal shape ratios
}

\author{
Hanbing Liu \\ College of Transportation, Jilin University, Changchun 130025, China \\ Email:1lhhbb@yahoo.cn \\ Yubo Jiao \\ College of Transportation, Jilin University, Changchun 130025, China \\ E-mail: jiaoyubo@yahoo.cn \\ Yafeng Gong \\ College of Transportation, Jilin University, Changchun 130025, China \\ E-mail: 423570235@qq.com \\ Received 10 May 2011 \\ Accepted 11 June 2012
}

\begin{abstract}
A fuzzy logic system (FLS) is established for damage identification of simply supported bridge. A novel damage indicator is developed based on ratios of mode shape components between before and after damage. Numerical simulation of a simply-supported bridge is presented to demonstrate the memory, inference and anti-noise ability of the proposed method. The bridge is divided into eight elements and nine nodes, the damage indicator vector at characteristic nodes is used as the input measurement of FLS. Results reveal that FLS can detect damage of training patterns with an accuracy of $100 \%$. Aiming at other test patterns, the FLS also possesses favorable inference ability, the identification accuracy for single damage location is up to $93.75 \%$. Tests with noise simulated data show that the FLS possesses favorable anti-noise ability.
\end{abstract}

Keywords: Damage identification; Simply-supported bridge; Fuzzy logic; Modal shape ratio

\section{Introduction}

There are totally 621.9 thousand highway bridges in China, small and medium span bridges are over 90\% according to the annual statistical report of Chinese highway maintenance [1]. These structures are susceptible to damage such as steel corrosion, concrete cracks and carbanation due to harsh environment, increasing traffic volume and material aging, their safety and durability performance degrade during service time. Therefore, it is essential to identify the damage of bridge in order to improve its operation efficiency [2-5].

Vibration-based damage identification method has attracted worldwide interest in the past few decades [6, 7]. The theoretical background is that the modal parameters (frequency, mode shape etc) are functions of physical properties (stiffness, mass etc) of bridge. The damage in structure alters its properties, and the modal parameters inevitably changed. The commonly used modal parameters for damage identification include natural frequency, mode shape and their derivatives, 
such as modal shape curvature, modal strain energy, modal flexibility etc [8-12].

Among these modal characteristics, natural frequency has no relation with measuring positions and can be measured most conveniently and accurately [6]. However, the frequency-based method possesses several apparent drawbacks. For instance, it is inferior sensitive to minor damage, and easily affected by environment [13]. Additionally, the simply supported reinforced concrete bridges are spatial symmetric structures. Natural frequency can not distinguish damage at symmetric locations in these symmetric structures.

These difficulties of using frequency as damage indicator can be overcome to some extent by using changes in mode shapes [14-16]. Comparing with natural frequency, mode shapes contain the spatial information with respect to location of damage. Meanwhile, they vary less sensitively to environmental effects. Modal perturbation analysis indicates that modal shape ratios are also less sensitive to the modeling errors than frequency [17].

Modal curvature is another most widely used damage indicator [10, 13, 14], which is the second spatial derivative of mode shape. It can be calculated by central difference approximation method in practice. But its calculation results possess apparent errors, which largely depend on the number of measuring points [18]. Modal shape ratios can be obtained more simply, which only need the mode shapes of structure before and after damage.

Artificial Neural Networks (ANN) have been utilized by many researchers to identify damage location and severity [17, 19-23], as they can achieve the nonlinear mapping between the inputs and outputs from training of certain amount of samples. But neural networks have the reputation of being black box that are difficult to understand [24]. Moreover, the test results of ANN are highly dependent on the accuracy of training samples. Fuzzy systems allow for easier understanding because they are expressed in terms of linguistic variables [25]. And they are finding increasing use in structural damage identification. Ganguli et al. [14, 2628] adopted the natural frequency and modal shape curvature as the input of fuzzy logic systems and genetic fuzzy logic systems for the damage detection of helicopter rotor blades. Zhao et al. [29] proposed a method based on principal component analysis, modified mountain clustering method, descent method and fuzzy logic systems for the damage detection of concrete bridges. Reda Taha [30] introduced a new techniques based on Bayesian updating and fuzzy sets to improve pattern recognition and damage detection of structures. However, the fuzzy logic method using mode shape ratio as input variables has not been reported.

In this paper, a novel damage indicator based on mode shape ratio is constructed. A simply supported bridge is divided into 8 elements with equal length and uniform bending rigidity. The damage indicator at several characteristic nodes is selected as the input measurements of FLS. The outputs of the FLS are damage levels of each element. Numerical simulation is conducted to verify the memory, inference and antinoise ability of the proposed method.

\section{Theoretical background}

\subsection{Theory of modal analysis}

The characteristic equations for vibration analysis of Euler-Bernoulli beam bridge can be written as[28, 31]

$$
\frac{\partial^{2}}{\partial x^{2}}\left[E I(x) \frac{\partial^{2} u(x, t)}{\partial x^{2}}\right]+m(x) \frac{\partial^{2} u(x, t)}{\partial t^{2}}=0
$$

where $E$ is the material elasticity of bridge, $I(x)$ is bending moment of inertia, $E I(x)$ is the flexural rigidity of the beam, $m(x)$ is mass per unit length of the beam and $u(x, t)$ is the transverse displacement of the beam reference axis. The beam equation is solved using the finite element method.

For an $n$ degree of freedom system, the equation of motion in discrete form is obtained after assembling of the element matrix and application of the boundary conditions.

$$
M \ddot{q}+K q=0
$$

here $M$ is the $n \times n$ mass matrix of the system, $K$ is the $n \times n$ rigidity matrix. $q$ is the $n \times 1$ vector of nodal displacements. We seek a solution of the form $q=\phi e^{(i w t)}$, which results in the eigenvalue problem.

$$
K \phi=\omega^{2} M \phi
$$

Solving this eigenvalue problem we get $n$ eigenvalues ( $\omega)$ and $n$ eigenvalues ( $\phi)$ which 
represent the natural frequencies and mode shapes of the system, respectively.

\subsection{Modeling of damage}

According to the theory of modal analysis, natural frequency and modal shape are closely related with rigidity ( $E I)$ and mass $(m)$ of bridge. In general, the damage of bridge does not alter its mass but the rigidity [17]. Therefore, the reduction of bridge rigidity caused by damage is the main factor leading to modal variation.

Taking the rectangular beam shown in Fig. 1 for example, $h$ is the section height, $b$ is the section width. The rigidity $k$ of this section can be expressed by

$$
k=E \cdot I=E \times \frac{b \times h^{3}}{12}
$$

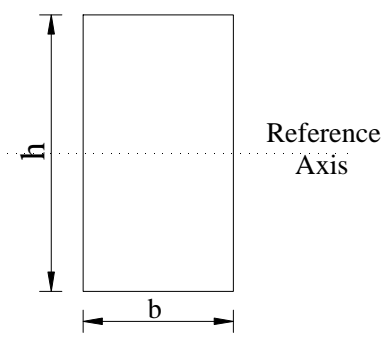

Fig. 1. Schematic diagram for rectangular section

As can be seen from Eq. (4), the rigidity of bridge contains two aspects that is elasticity and section size ( $b$ and $h$ ). In the present research, there are usually two simulation method for damage, one assumes that the section size of bridge is changed [32, 33], while the other assumes that the elasticity of bridge material is altered [17, 24, 28]. In this paper, the latter method is adopted, the damage parameter $\alpha_{i}$ is defined by

$$
\alpha_{i}=1-E_{i, d} / E_{i, u}
$$

where $i$ is the number of damaged element, $E_{i, u}, E_{i, d}$ represent elasticity of undamaged and damaged element, respectively.

A numerical example analysis is performed on a simply supported bridge as shown in Fig. 2. The model consists of 8 elements with equal element length $\left(\mathrm{L}^{\mathrm{EL}}=1 \mathrm{~m}\right)$ and uniform bending rigidity $\left(E I=1.155 \times 10^{7} \mathrm{kN} . \mathrm{m}^{2}\right)$ for undamaged structure.

\begin{tabular}{|l|l|l|l|l|l|l|l|}
\hline & 2 & \multicolumn{1}{l}{3} & \multicolumn{1}{l}{4} & 5 & 6 & 7 & 8 \\
\hline 1 & 2 & 3 & 4 & 5 & 6 & 7 & 8 \\
\hline
\end{tabular}

Fig. 2. Model of simply supported bridge

In this paper, the structural damage in each element is simulated by elasticity reduction $\left(\alpha_{i}\right)$ of $5,10,15$ percent for method validation. These damages are classified as "slight damage", "moderate damage", "severe damage”, respectively. Damage sizes below "slight damage" are classified as "undamaged" and damage sizes greater than "severe damage" are classified as "catastrophic damage".

In practical engineering, the selected $\alpha_{i}$ for "slight damage", "moderate damage", "severe damage" can be adjusted in accordance with the span, load level and importance for transportation of bridge. Meanwhile, According to the identified damage condition, appropriate maintenance strategy may be adopted by engineers in order to ensure the safe operation of bridge.

\subsection{Fuzzy logic system}

Fuzzy logic system is a computational mechanism based on fuzzy set, fuzzy rules and fuzzy inference. It can achieve the nonlinear mapping between inputs and outputs [25, 30]. Mamdani fuzzy system is the most widely used one, it consists of fuzzification of input measurements, fuzzy rules, fuzzy inference and defuzzification of output measurements as shown in Fig. 3 [14, 24].

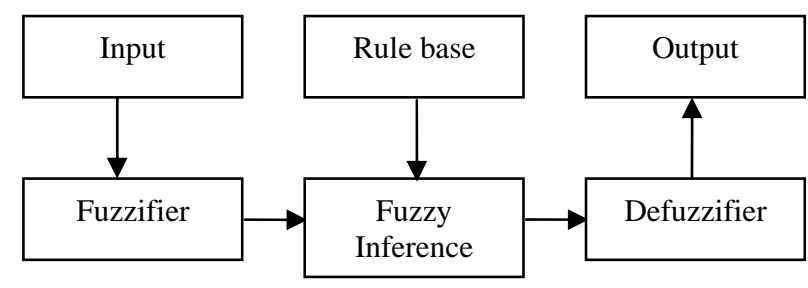

Fig. 3. Schematic representation of Mamdani system

\subsubsection{Input and output}

The point of fuzzy logic is to map an input vector represented by $x$ to an output one represented by $y$. Mathematically, this can be represented as [14]

$$
y=f(x)
$$


In this paper, a novel measurement based on ratios of mode shape components between before and after damage is constructed as the input of FLS. The mode shape ratio is defined by

$$
\overline{\Delta \phi^{m}{ }_{n}}=\phi^{m}{ }_{d n} / \phi^{m}{ }_{u n}
$$

where $m$ is the order of mode shape, $n$ is the number of nodes, $\phi_{d n}^{m}, \phi^{m}$ un represent the $m$ th mode shape value at node $n$ for damaged and undamaged structure, respectively.

Taking damage identification of element 4 with damage severity $10 \%$ for example, the first modal shapes $(m=1)$ for all nodes of bridge under damaged and undamaged condition are listed in Table 1 and Fig.4. It can be concluded the modal shapes at nodes have changed, this reveals that the bridge has been damaged.

Table 1 First modal shapes of damaged and undamaged bridge

\begin{tabular}{ccc}
\hline \multirow{2}{*}{$\begin{array}{c}\text { Node } \\
\text { Number }\end{array}$} & \multicolumn{2}{c}{ Element 4 with damage severity } \\
\cline { 2 - 3 } & Undamaged & Damaged \\
\hline 1 & 0 & 0 \\
2 & 0.0040917 & 0.0040851 \\
3 & 0.0075604 & 0.0075630 \\
4 & 0.0098781 & 0.0099189 \\
5 & 0.0106920 & 0.0107220 \\
6 & 0.0098781 & 0.0098514 \\
7 & 0.0075604 & 0.0075160 \\
8 & 0.0040917 & 0.0040609 \\
9 & 0 & 0 \\
\hline
\end{tabular}

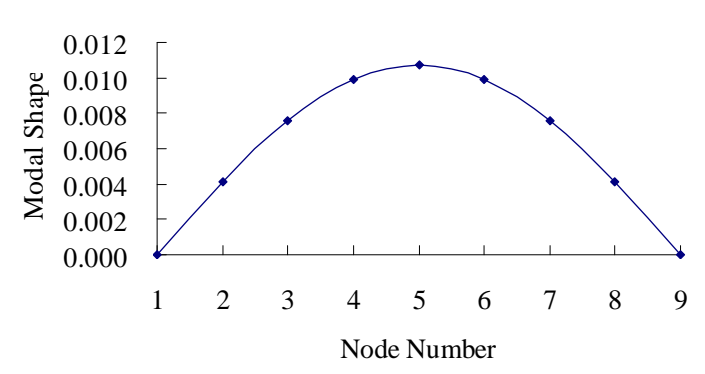

Fig. 4. Modal shapes for undamaged bridge

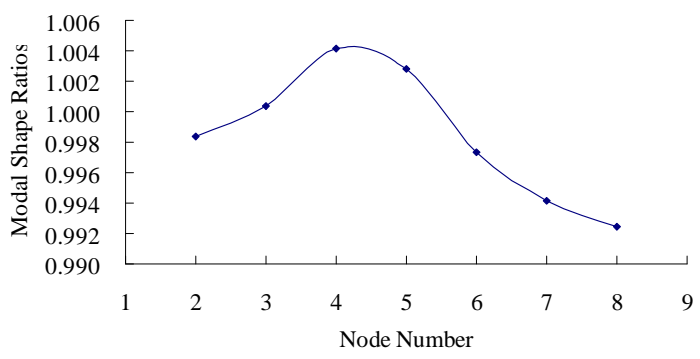

Fig. 5. Modal shape ratios between before and after damage

The mode shape ratios calculated by Eq. (7) without considering fulcrum (nodes 1 and 9) are shown in Fig. 5. As can be seen from Fig. 5., modal shape ratios emerge a peak at the relevant nodes (nodes 4 and 5) of element 4 , which can be used to identify the damage location. It indicates that mode shape ratio is a favorable damage indicator.

We can also find that the values of mode shape ratios are all around number " 1 ", and their interval range is not obvious. That is not conducive to establish the FLS. Therefore, the mode shape ratios are furtherly transformed as follows.

$$
\left.\Delta \phi_{n}^{m}=\mid \overline{\Delta \phi^{m}{ }_{n}} * 1000-1000\right) \mid
$$

where $\Delta \phi^{m}{ }_{n}$ is the damage indicator used in this paper, $\overline{\Delta \phi^{m}}$ is the mode shape ratio.

The mode shape ratios before and after transformation are listed in Table 2.

\begin{tabular}{|c|c|c|}
\hline \multirow{2}{*}{$\begin{array}{l}\text { Node } \\
\text { Number }\end{array}$} & \multicolumn{2}{|c|}{$\begin{array}{c}\text { Element } 4 \text { with damage severity } \\
10 \%\end{array}$} \\
\hline & $\Delta \phi^{m}{ }_{n}$ & $\Delta \phi^{m}{ }_{n}$ \\
\hline 2 & 0.9984 & 1.6130 \\
\hline 3 & 1.0003 & 0.3439 \\
\hline 4 & 1.0041 & 4.1303 \\
\hline 5 & 1.0028 & 2.8058 \\
\hline 6 & 0.9973 & 2.7029 \\
\hline 7 & 0.9941 & 5.8727 \\
\hline 8 & 0.9925 & 7.5274 \\
\hline
\end{tabular}

Table 2 Mode shape ratios before and after transformation

As can be seen from Table 2, the range of $\Delta \phi^{m}{ }_{n}$ is more obvious, which is convenient for the construction of FLS. 
Structural damage severity $\left(\alpha_{i}\right)$ is used as the output of the FLS. Therefore, the objective of FLS is to find a functional mapping between $\Delta \phi^{m}{ }_{n}$ and $\alpha_{i}$.

\subsubsection{Fuzzification}

The fuzzification comprises the process of transforming crisp values into grades of membership for linguistic terms of fuzzy sets. The membership function is used to associate a grade to each linguistic term [34].

Inputs to the fuzzy logic system are the measurements of $\Delta \phi^{m}{ }_{n}$ and outputs are structural damage degree $\left(\alpha_{i}\right)$ of elements. For input variables, we define nine membership functions to transform $\Delta \phi^{m}{ }_{n}$ into linguistic terms of fuzzy sets.

$T\left(\Delta \phi_{n}^{m}\right)=$ \{negligible, very low, low, low medium, medium, medium high, high, very high, very very high $\}$

For output variables, we define five membership functions to transform the damage degree into linguistic terms of fuzzy sets. For example, "element $i$ ( $i=1,2, \ldots, 8)$ " is considered as a linguistic variable. It is decomposed into a set of terms.

$T$ (element $i$ ) $=$ undamaged, slight damage, moderate damage, severe damage, catastrophic damage\}, $i=1,2, \ldots, 8$.

Fuzzy sets with gaussian membership functions are used for the input variables. These fuzzy sets can be defined using the following equation.

$$
\mu(x)=e^{-0.5((x-m) / \sigma)^{2}}
$$

where $m$ is the midpoint of the fuzzy set and $\sigma$ is the standard deviation associated with the variables. The midpoints are selected to span the region ranging from an undamaged bridge to one with significant damage, while the standard deviations are selected in order to guarantee the enough width to capture the variations of the input variables. Therefore, the reasonable selection of $m$ and $\sigma$ is very important for the performance of the FLS.

Fuzzy sets with bell functions are used for the output variables. These fuzzy sets can be defined using the following equation.

$$
\mu(y)=\frac{1}{1+\left|\frac{y-c}{a}\right|^{2 b}}
$$

where [a, b, c] are the variables that control the shape of the function.

\subsubsection{Fuzzy rules and its generation}

Rules for fuzzy system can be expressed as: $R^{i}:$ if $\Delta \phi^{m}{ }_{1}$ is $P_{1}, \Delta \phi^{m}{ }_{2}$ is $P_{2} \ldots \Delta \phi^{m}{ }_{n}$ is $P_{n}$, then $\alpha$ is $Q_{i}, \quad i=1,2, \ldots, q$

where $R^{i}$ denotes the $i$ th rule, $\Delta \phi^{m}{ }_{n}$ is mode shape ratio and input variable, $\alpha$ represents damage severity of elements and used as the output variable, $n, q$ are the number of input and output variables, $P$ and $Q$ are the fuzzy sets with the membership function $\mu(x)$, $\mu(y)$ as defined in Eqs. (9) and (10).

Rules for the fuzzy system are obtained by fuzzification of the numerical values obtained from finite element analysis using the following procedure:

- The damage indicator $\Delta \phi^{m}{ }_{n}$ corresponding to a given damage severity is input into the FLS and the membership degree of $\Delta \phi^{m}{ }_{n}$ is obtained. Therefore, each measurement has nine degree of memberships based on the linguistic measures.

- Each measurement variable is then assigned to the fuzzy set with the maximum degree of membership.

- One rule is obtained for each damage severity by relating the measurement variable with maximum degree of membership to a fault.

\subsubsection{Defuzzification}

Defuzzification is interpreting the membership degrees of fuzzy sets into a specific decision or real value. In order to obtain the damage state of elements, the outputs must be defuzzified. There are many different methods of defuzzification available, such as LOM (last of maximum), COA (center of area), COG (center of gravity), FCD (fuzzy clustering defuzzification) etc [35].

In this paper, the LOM method is chosen as the defuzzification strategy, which is easy for bridge engineers to master and apply. This method gives the output with the highest membership function. It can be expressed by

$$
\alpha_{0}=d f(\alpha)=d f\left(\alpha_{u d}, \alpha_{s d}, \alpha_{m d}, \alpha_{s e d}, \alpha_{c d}\right)
$$

where $d f$ is the defuzzification function, $\alpha_{0}$ is the identified damage condition after defuzzification, $\alpha$ is the vector of membership degree for all damage conditions, such as "undamaged ( $\left.\alpha_{u d}\right)$ ", "slight damage $\left(\alpha_{s d}\right)$ ", "moderate damage $\left(\alpha_{m d}\right)$ ", "severe 
damage $\left(\alpha_{\text {sed }}\right)$ ), “catastrophic damage $\left(\alpha_{c d}\right)$ ”. $\alpha_{0}$ can be determined according to the damage condition with maximum membership degree.

\subsection{Modeling of uncertainty}

Uncertainties inevitably exist in experimental measurements due to modeling and measurement uncertainties. The damage indicator with noise can be obtained after adding measurement noise into the calculated indicator [36].

$$
\Delta \phi_{\text {noise }}=\Delta \phi(1+\lambda \times \text { normrnd }(0,1))
$$

here $\Delta \phi_{\text {noise }}$ is the damage indicator with noise, while $\Delta \phi$ is the indicator without noise, $\lambda$ is the noise level parameter, normrnd $(0,1)$ is gaussian random number.

\section{Numerical simulation}

\subsection{FLS for damage identification of simply supported bridge}

\subsubsection{Input and output variables}

In general, the damage indicator at all nodes except fulcrum is used as the input variable. But there are several drawbacks for this method. Firstly, it is not necessary, because mode shape ratios at partial nodes already contain enough damage information. Secondly, there will be data loss in practical measurement, we can not obtain the information at all nodes. Therefore, the damage indicator at the nodes 2, 4, 5, 6, 8 of the first mode shape is selected as the input variables in this paper, which is shown in Fig. 6. The output variable is the damage severity of elements.

input $=\left\{\Delta \Phi^{1}{ }_{2}, \Delta \Phi_{4}^{1}, \Delta \Phi^{1}{ }_{5}, \Delta \Phi_{6}^{1}, \Delta \Phi^{1}{ }_{8}\right\}$

output $=$ \{element 1 , element 2 , element 3 , element 4 , element 5 , element 6 , element 7 , element 8 \}

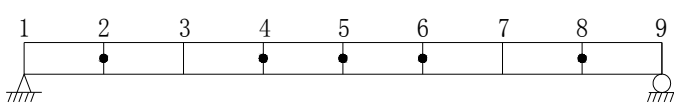

Fig. 6. Characteristic nodes of mode shape for fuzzy system

\subsubsection{Damage identification cases}

The damage cases are established to verify the effectiveness of FLS as shown in Table 3.

Table 3 Cases for damage identification

\begin{tabular}{cccc}
\hline Case No. & 1 & 2 & 3 \\
\hline $\begin{array}{l}\text { Damaged } \\
\text { elements }\end{array}$ & Element 1 & Element 4 & Element 4, 5 \\
\hline
\end{tabular}

\subsubsection{Membership functions for inputs and outputs}

Fuzzy sets with gaussian membership functions are used for the input variables, different midpoints $(m)$ and standard deviations $(\sigma)$ are selected for different cases in order to reduce the repetition of rules and optimize the accuracy of detection. Fuzzy sets with bell function are used for the output variables, the same membership function is used for all cases. The membership functions of inputs and outputs are shown in Fig. 7., Fig. 8. and Table 4.

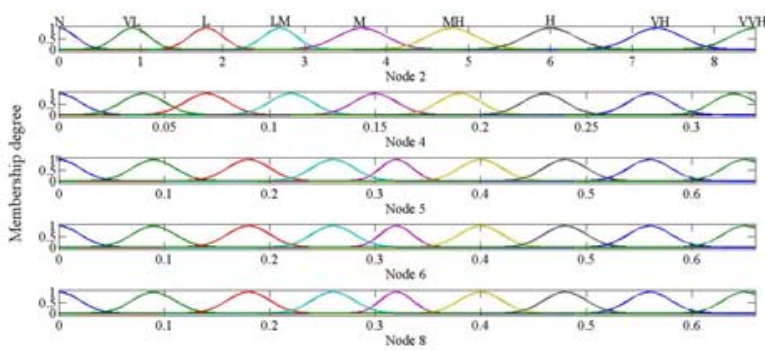

(a) Membership functions of case 1

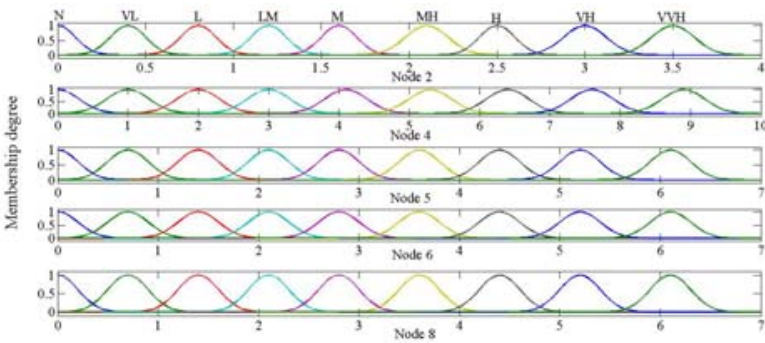

(b) Membership functions of case 2

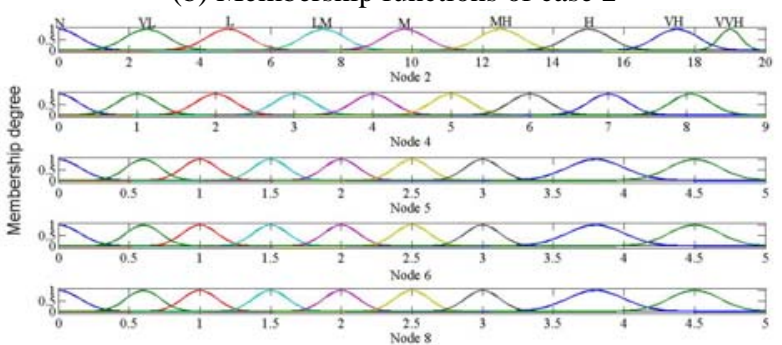

(c) Membership functions of case 3

Fig. 7. Schematic diagram of membership function of inputs 
Table 4 Fuzzy sets for output measurements

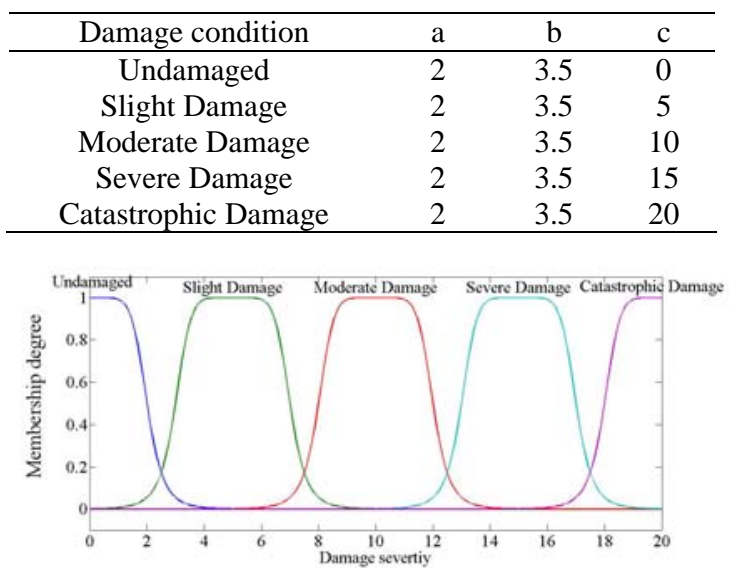

Fig. 8. Schematic diagram of membership function of outputs

In order to make the expression more concise, linguistic measures of inputs and outputs are expressed by corresponding simplified symbols as shown in Tables 5 and 6.

Table 5 Symbols of fuzzy variables of inputs

\begin{tabular}{cc}
\hline Linguistic measure & Symbol \\
\hline Negligible & $\mathrm{N}$ \\
Very Low & $\mathrm{VL}$ \\
Low & $\mathrm{L}$ \\
Low-Medium & $\mathrm{LM}$ \\
Medium & $\mathrm{M}$ \\
Medium-High & $\mathrm{MH}$ \\
High & $\mathrm{H}$ \\
Very High & $\mathrm{VH}$ \\
Very Very High & $\mathrm{VVH}$ \\
\hline
\end{tabular}

Table 6 Symbols of fuzzy variables of outputs

\begin{tabular}{cl}
\hline Linguistic measure & Symbol \\
\hline Undamaged & UD (a) \\
Slight Damage & SD (b) \\
Moderate Damage & MD (c) \\
Severe Damage & SED (d) \\
Catastrophic Damage & CD (e) \\
\hline
\end{tabular}

\subsubsection{Fuzzy rule bases}

According to the rules generation method listed in section 2.3.3, fuzzy rule bases of cases 1, 2 and 3 are listed in Tables 7, 8 and 9.

The fuzzy rule of "element 4 with moderate damage and element 5 with severe damage" can be expressed as:

If $\Delta \Phi^{1}{ }_{2}$ is " very high "; and $\Delta \Phi^{1}{ }_{4}$ is " negligible "; and $\Delta \Phi^{1}{ }_{5}$ is " medium high "; and $\Delta \Phi^{1}{ }_{6}$ is "medium high "; and $\Delta \Phi^{1}{ }_{8}$ is "medium high ";

Then "element 4 with moderate damage and element 5 with severe damage”.

The other fuzzy rules in Tables 7, 8 and 9 can be interpreted in the same way.

It can be seen from the tables that as the damage becomes more severe, the damage indicator changes from "low” to "medium” and "high” levels.

As indicated from Tables 7, 8 and 9, each rule represents a unique damage situation and is different from all the other rules. Therefore the FLS is a good pattern classifier, it is applicable to damage identification of bridges. These rules provide a knowledge base and represent how an engineer would interpret data to isolate structural damage using indicators proposed in this paper.

Table 7 Fuzzy rules of case 1

\begin{tabular}{cccccc}
\hline Damage & \multicolumn{5}{c}{ Inputs } \\
\cline { 2 - 6 } levels & $\Delta \Phi^{1}{ }_{2}$ & $\Delta \Phi^{1}{ }_{4}$ & $\Delta \Phi^{1}{ }_{5}$ & $\Delta \Phi^{1}{ }_{6}$ & $\Delta \Phi^{1}{ }_{8}$ \\
\hline 1-UD & $\mathrm{N}$ & $\mathrm{N}$ & $\mathrm{N}$ & $\mathrm{N}$ & $\mathrm{N}$ \\
1-SD & $\mathrm{L}$ & $\mathrm{L}$ & $\mathrm{VL}$ & $\mathrm{L}$ & $\mathrm{L}$ \\
1-MD & $\mathrm{M}$ & $\mathrm{M}$ & $\mathrm{LM}$ & $\mathrm{M}$ & $\mathrm{M}$ \\
1-SED & $\mathrm{H}$ & $\mathrm{H}$ & $\mathrm{H}$ & $\mathrm{H}$ & $\mathrm{H}$ \\
1-CD & $\mathrm{VVH}$ & $\mathrm{VVH}$ & $\mathrm{VVH}$ & $\mathrm{VVH}$ & $\mathrm{VVH}$ \\
\hline
\end{tabular}

Table 8 Fuzzy rules of case 2

\begin{tabular}{cccccc}
\hline \multirow{2}{*}{$\begin{array}{c}\text { Damage } \\
\text { levels }\end{array}$} & $\Delta \Phi^{1_{2}}$ & $\Delta \Phi^{1}{ }_{4}$ & $\Delta \Phi^{1}$ & $\Delta \Phi^{1}{ }_{6}$ & $\Delta \Phi^{{ }^{1}} 8$ \\
\cline { 2 - 6 } & $\mathrm{N}$ & $\mathrm{N}$ & $\mathrm{N}$ & $\mathrm{N}$ & $\mathrm{N}$ \\
4-UD & $\mathrm{L}$ & $\mathrm{L}$ & $\mathrm{L}$ & $\mathrm{L}$ & $\mathrm{L}$ \\
4-SD & $\mathrm{M}$ & $\mathrm{M}$ & $\mathrm{M}$ & $\mathrm{M}$ & $\mathrm{M}$ \\
4-MD & $\mathrm{H}$ & $\mathrm{H}$ & $\mathrm{H}$ & $\mathrm{H}$ & $\mathrm{H}$ \\
4-SED & $\mathrm{VVH}$ & $\mathrm{VVH}$ & $\mathrm{VVH}$ & $\mathrm{VVH}$ & $\mathrm{VVH}$ \\
4-CD & & & & &
\end{tabular}


Table 9 Fuzzy rules of case 3

\begin{tabular}{|c|c|c|c|c|c|}
\hline \multirow{2}{*}{ Damage levels } & \multicolumn{5}{|c|}{ Inputs } \\
\hline & $\Delta \Phi^{1}{ }_{2}$ & $\Delta \Phi^{1}{ }_{4}$ & $\Delta \Phi^{1}$ & $\Delta \Phi_{6}^{1}$ & $\Delta \Phi_{8}^{1}$ \\
\hline All-UD & $\mathrm{N}$ & $\mathrm{N}$ & $\mathrm{N}$ & $\mathrm{N}$ & $\mathrm{N}$ \\
\hline 4-UD, 5-SD & $\mathrm{L}$ & $\mathrm{L}$ & VL & LM & $\mathrm{N}$ \\
\hline 4-UD, 5-MD & M & M & $\mathrm{L}$ & $\mathrm{H}$ & VL \\
\hline 4-UD, 5-SED & $\mathrm{H}$ & $\mathrm{H}$ & LM & $\mathrm{VVH}$ & VL \\
\hline 4-SD, 5-UD & $\mathrm{N}$ & LM & VL & $\mathrm{L}$ & $\mathrm{L}$ \\
\hline 4-SD, 5-SD & $\mathrm{L}$ & VL & $\mathrm{L}$ & VL & $\mathrm{L}$ \\
\hline 4-SD, 5-MD & M & VL & LM & M & LM \\
\hline 4-SD, 5-SED & $\mathrm{H}$ & LM & M & $\mathrm{VH}$ & LM \\
\hline 4-MD, 5-UD & VL & $\mathrm{H}$ & $\mathrm{L}$ & M & M \\
\hline 4-MD, 5-SD & LM & M & LM & VL & M \\
\hline 4-MD, 5-MD & MH & $\mathrm{L}$ & M & $\mathrm{L}$ & $\mathrm{MH}$ \\
\hline 4-MD， 5-SED & VH & $\mathrm{N}$ & MH & $\mathrm{MH}$ & MH \\
\hline 4-SED, 5-UD & VL & VVH & LM & $\mathrm{H}$ & $\mathrm{H}$ \\
\hline 4-SED, 5-SD & LM & $\mathrm{VH}$ & M & LM & $\mathrm{H}$ \\
\hline 4-SED, 5-MD & $\mathrm{MH}$ & $\mathrm{MH}$ & $\mathrm{MH}$ & $\mathrm{N}$ & $\mathrm{VH}$ \\
\hline 4-SED, 5-SED & $\mathrm{VH}$ & LM & $\mathrm{H}$ & LM & $\mathrm{VH}$ \\
\hline
\end{tabular}

\subsection{Results and discussion}

To verify the effectiveness of FLS proposed in this paper, its memory, inference and anti-noise ability are examined. The purpose is to guarantee that the FLS using mode shape ratio as input variable possesses satisfactory robustness and generalization ability. In this paper, the training and test samples are obtained through finite element analysis and calculation.

\subsubsection{Memory ability of FLS}

In order to verify the memory ability of FLS, 26 training patterns of cases 1, 2 and 3 are used as the inputs of FLS. Numerical simulation shows that the proposed method in this paper could identify the damage with an accuracy of $100 \%$. The identification results of case 1 and 3 are shown in Tables 10 and 11.

Table 10 Memory ability of fuzzy system for case 1

\begin{tabular}{c|ccccc|ccccc}
\hline Nodes & \multicolumn{5}{|c|}{ Ideal outputs } & \multicolumn{5}{c}{ Actual outputs } \\
\hline 2 & 1 & 0 & 0 & 0 & 0 & 0.99 & 0 & 0 & 0 & 0 \\
4 & 0 & 1 & 0 & 0 & 0 & 0.01 & 1 & 0 & 0 & 0 \\
5 & 0 & 0 & 1 & 0 & 0 & 0 & 0 & 1 & 0 & 0 \\
6 & 0 & 0 & 0 & 1 & 0 & 0 & 0 & 0 & 1 & 0.01 \\
8 & 0 & 0 & 0 & 0 & 1 & 0 & 0 & 0 & 0 & 0.97 \\
\hline
\end{tabular}

\subsubsection{Inference ability of FLS}

The following damage circumstances listed in Table 12 are established to verity the inference ability of FLS proposed in this paper. Case 2 is used to demonstrate the inference ability of FLS with single damage location, while case 3 for the multiple damage locations. In Table 12 , the expression " $14 \%-4 \%$ " is representative of "damage severity is $14 \%$ for element 4 , while $4 \%$ for element 5 ", the other expressions are the same representations.

Damage identification results of case 2 with damage degree $8 \%$ and $14 \%$ are shown in Fig. 9. It is shown that the damage levels of element 4 are "moderate damage" and "severe damage", respectively. If the damage degree $8 \%$ is fuzzified by membership function of outputs as shown in Fig. 8., its linguistic variable is "moderate damage", while damage degree $14 \%$ is "severe damage". Therefore, the FLS have a correct damage identification results. As for other circumstances in Table 12, the FLS can identify all the damage degrees except $17 \%$, the identification accuracy is $93.75 \%$. The numerical simulation shows that the FLS possess favorable inference ability for damage identification with single location.

The actual identification results of case 3 with multiple damage locations are shown in Fig. 10. The damage degrees are fuzzified by membership function, 
and the ideal results are listed in Table 13. Contrasting analysis demonstrated that the FLS possessed favorable inference ability for damage identification with multiple locations.

Table 11 Memory ability of fuzzy system for case 3

\begin{tabular}{ccccc}
\hline \multirow{2}{*}{$\begin{array}{c}\text { Damage } \\
\text { level }\end{array}$} & \multicolumn{2}{c}{$\begin{array}{c}\text { Element 4 with "slight damage" } \\
\text { Element 5 with "moderate damage" }\end{array}$} & \multicolumn{2}{c}{$\begin{array}{c}\text { Element 4 with "moderate damage" } \\
\text { Element 5 with "severe damage" }\end{array}$} \\
\cline { 2 - 5 } & Outputs of element4 & Outputs of element 5 & Outputs of element4 & Outputs of element 5 \\
\hline "Undamaged" & 0.002 & 0 & 0 & 0 \\
"Slight" & 1 & 0.002 & 0.002 & 0 \\
"Moderate" & 0.002 & 1 & 1 & 0.002 \\
"Severe" & 0 & 0.002 & 0.002 & 1 \\
"Catastrophic" & 0 & 0 & 0 & 0.002 \\
\hline
\end{tabular}

Table 12 Damage cases for inference ability of fuzzy systems

\begin{tabular}{ccc}
\hline Circumstances & Damage identification with single location (case 2) & Damage identification with multiple locations (case 3) \\
\hline Damage & $1 \% ; 2 \% ; 3 \% ; 4 \% ; 6 \% ; 7 \% ; 8 \% ; 9 \% ; 11 \% ;$ & $14 \%-4 \% ; 6 \%-6 \%$ \\
Severity & $12 \% ; 13 \% ; 14 \% ; 16 \% ; 17 \% ; 18 \% ; 19 \%$ & $8 \%-12 \% ; 12 \%-6 \%$ \\
\hline
\end{tabular}

Table 13 Identification of damage with multiple locations

\begin{tabular}{|c|c|c|}
\hline $\begin{array}{l}\text { Identification } \\
\text { results }\end{array}$ & $\begin{array}{c}\text { Damage } \\
\text { degree }\end{array}$ & Damage levels \\
\hline \multirow{4}{*}{ Actual outputs } & $14 \%-4 \%$ & $\begin{array}{l}\text { Element } 4 \text { "severe damage”; } \\
\text { Element } 5 \text { “moderate damage” }\end{array}$ \\
\hline & $6 \%-6 \%$ & $\begin{array}{l}\text { Element } 4 \text { “slight damage”; } \\
\text { Element } 5 \text { “slight damage” }\end{array}$ \\
\hline & $8 \%-12 \%$ & $\begin{array}{l}\text { Element } 4 \text { "moderate damage"; } \\
\text { Element } 5 \text { "moderate damage" }\end{array}$ \\
\hline & $12 \%-6 \%$ & $\begin{array}{c}\text { Element } 4 \text { “moderate damage”; } \\
\text { Element } 5 \text { "slight damage” }\end{array}$ \\
\hline \multirow{4}{*}{ Ideal outputs } & $14 \%-4 \%$ & $\begin{array}{l}\text { Element } 4 \text { "severe damage”; } \\
\text { Element } 5 \text { “moderate damage" }\end{array}$ \\
\hline & 6\%-6\% & $\begin{array}{l}\text { Element } 4 \text { “slight damage”; } \\
\text { Element } 5 \text { “slight damage” }\end{array}$ \\
\hline & $8 \%-12 \%$ & $\begin{array}{l}\text { Element } 4 \text { "moderate damage"; } \\
\text { Element } 5 \text { "moderate damage" }\end{array}$ \\
\hline & $12 \%-6 \%$ & $\begin{array}{c}\text { Element } 4 \text { "moderate damage"; } \\
\text { Element } 5 \text { "slight damage" }\end{array}$ \\
\hline
\end{tabular}

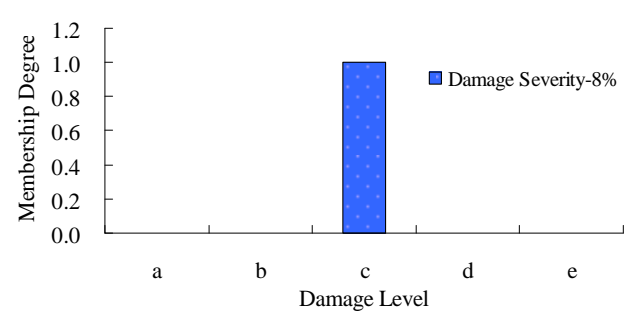

(1) Identification of damage severity $8 \%$

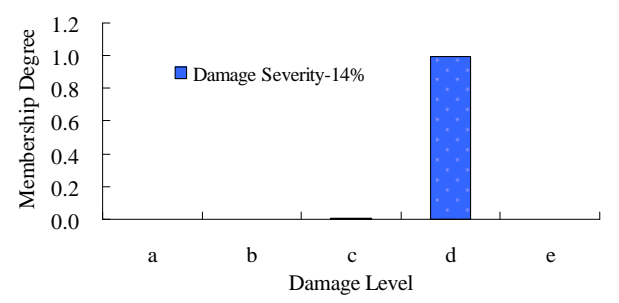

(2) Identification of damage severity $14 \%$

Fig. 9. Damage identification of case 2 


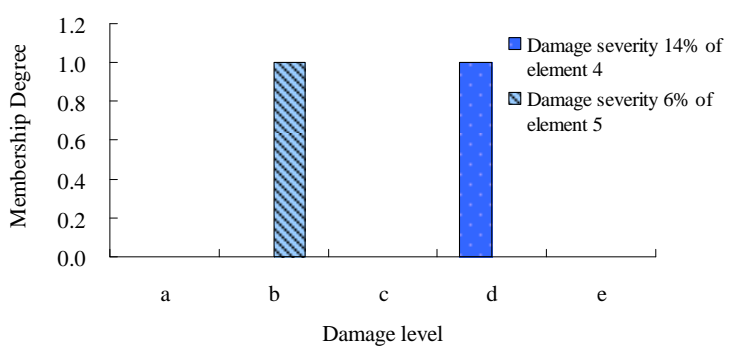

(1) Identification of damage severity $14 \%-6 \%$

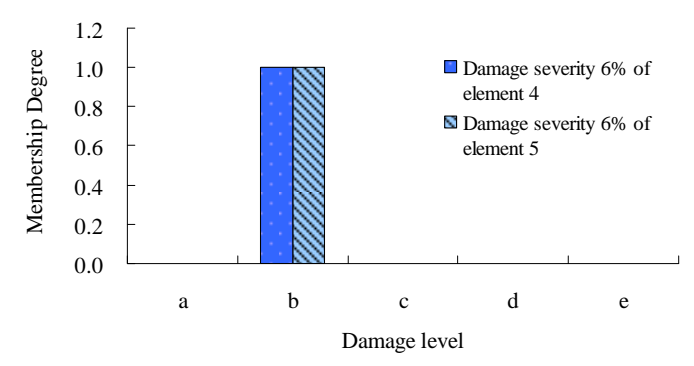

(2) Identification of damage severity 6\%-6\%

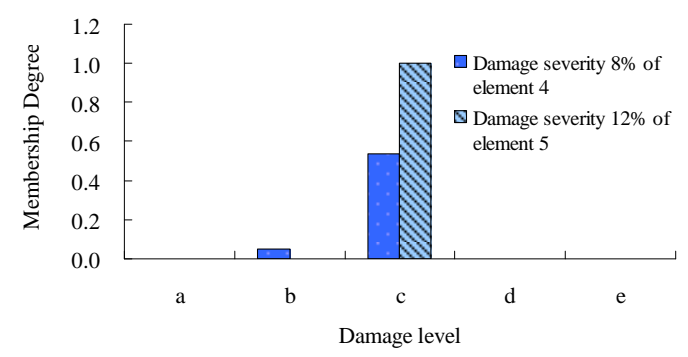

(3) Identification of damage severity $8 \%-12 \%$

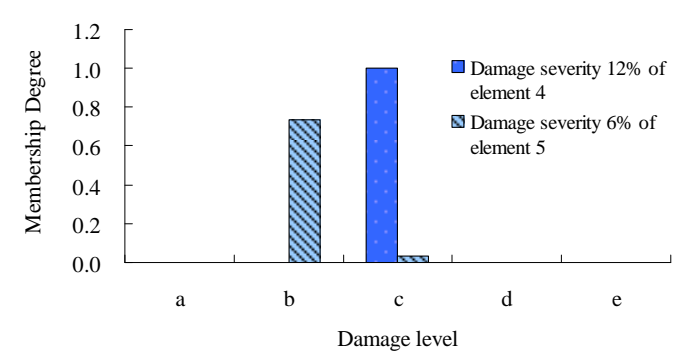

(4) Identification of damage severity $12 \%-6 \%$

Fig. 10. Damage identification of case 3 obtained according to the maximum membership degree. As can be seen from these figures, FLS can identify the damage under the noise level $50 \%$ for the degree $7 \%$, while $20 \%$ for the degree $15 \%$. It demonstrated that the FLS proposed in this paper possesses satisfactory antinoise ability for the damage identification of single location.

Case 3 with damage degree 10\%-15\% and 12\%-6\% are used to verify the anti-noise ability of FLS for the damage identification with multiple locations. The results are demonstrated in Figs. 13 and 14. As for the damage degree $10 \%-15 \%$, FLS can identify the damage under noise level $30 \%$, While $15 \%$ for the damage degree $14 \%-4 \%$. Numerical simulation shown that the FLS proposed in this paper possess favorable anti-noise ability for the damage identification with multiple locations.

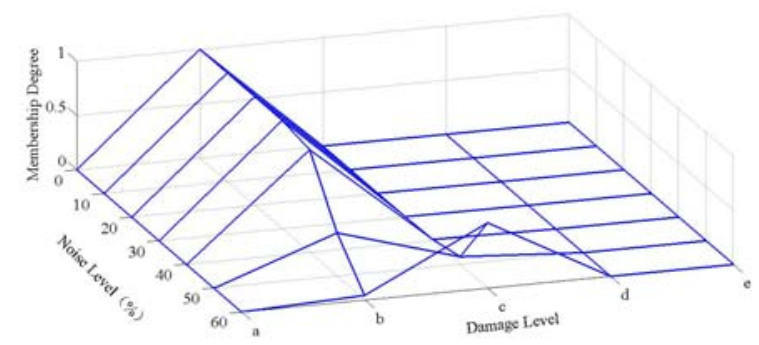

Fig. 11. Anti-noise analysis of element 4 with damage degree $7 \%$

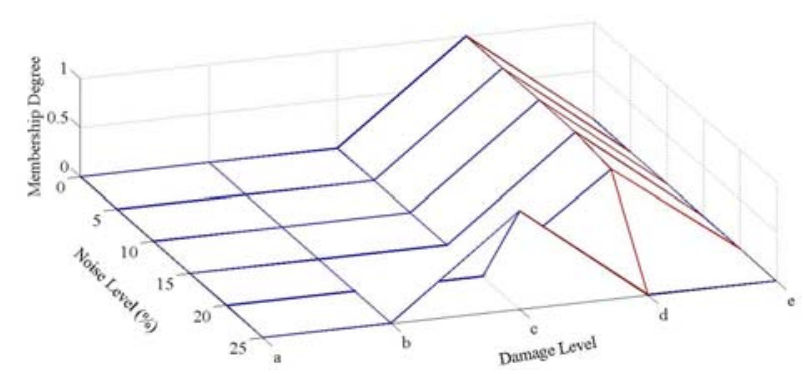

Fig. 12. Anti-noise analysis of element 4 with damage degree $15 \%$

\subsubsection{Anti-noise ability of FLS}

The identification of case 2 with damage degree $7 \%$ and $15 \%$ is used to verify the anti-noise ability of FLS proposed in this paper for the damage identification with single location. The identification results are shown in Figs. 11 and 12. The damage level can be 

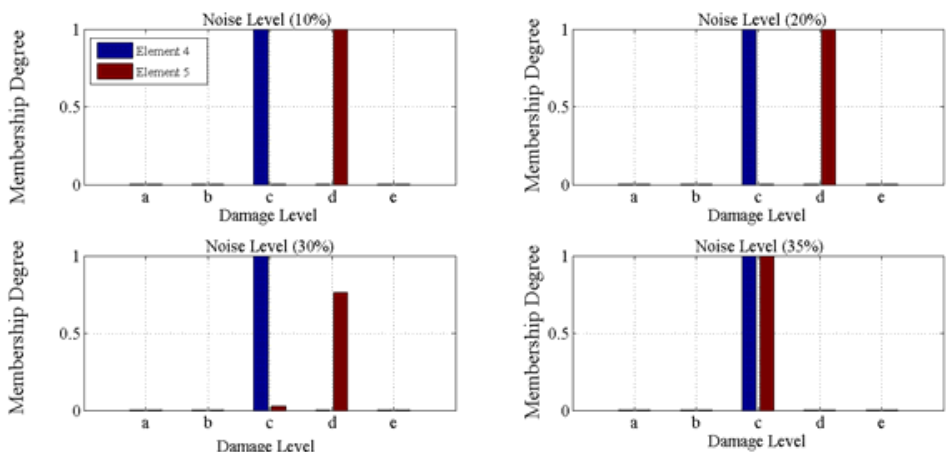

Fig. 13. Damage identification of case 3 with damage degree $10 \%-15 \%$
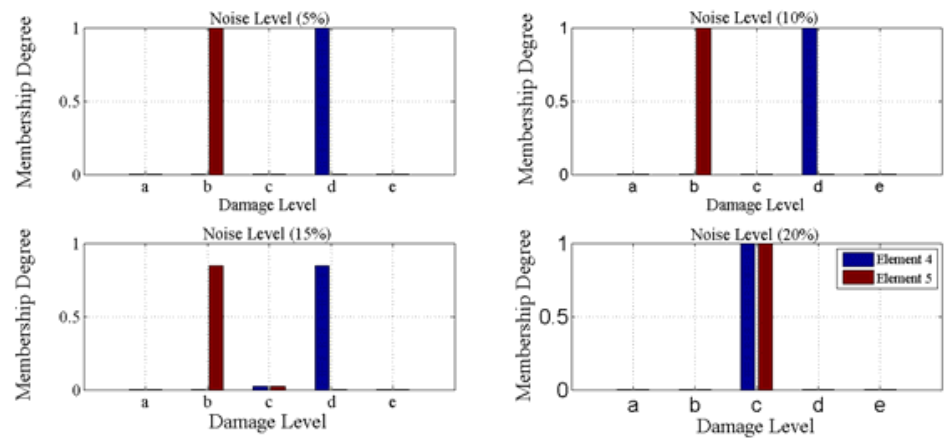

Fig. 14. Damage identification of case 3 with damage degree $14 \%-4 \%$

\section{Conclusions}

We have demonstrated a method to identify the damage of simply supported bridges based on FLS. A novel damage indicator transformed from mode shape ratios is proposed in this paper and used as the input measurement of FLS, while damage severity of element is treated as the ouput one. Gauss and Bell membership functions are selected for fuzzification of input and output variables, respectively.

Numerical simulation on selected damage cases is conducted to verify the memory, inference and antinoise ability of the proposed method. As for the memory ability, FLS can detect damage of training patterns with an accuracy of $100 \%$ for single and multiple damage locations. Aiming at other test patterns, the FLS also possesses favorable inference ability, the identification accuracy for single damage location is up to $93.75 \%$. Tests with noise simulated data show that the FLS can detect damage very accurately at different noise levels.

\section{Acknowledgments}

The authors gratefully acknowledge the support of the National High Technology Research and Development Program ("863 program") of China (Project No. 2009AA11Z104), "985 Project” of Jilin University and the support of innovation team program of Jilin university.

\section{References}

1. Annual report of Chinese highway maintenance statistical report, 2009. (In Chinese)

2. Y.M. Wang, T.M.S. Elhag, Evidential reasoning approach for bridge condition assessment, Expert Syst Appl. 34 (1) (2008) 689-699.

3. S. Sasmal, K. Ramanjaneyulu, S. Gopalakrishnan, N. Lakshmanan, Fuzzy logic based condition rating of existing reinforced concrete bridge, $J$ PERFORM CONSTR FAC. 20 (3) (2006) 261-273. 
4. A. Tarighat, A. Miyamoto, Fuzzy concrete bridge deck condition rating method for practical bridge management systems, Expert Syst Appl. 36 (10) (2009) 12077-12085.

5. S.W. Doebling, C.R. Farrar, M.B Prime, et al. A review of damage identification methods that examine changes in dynamic properties, SHOCK VIB Dig. 30 (2) (1998) 91-105.

6. O.S. Salawu, Detection of structural damage through changes in frequency: A review, ENG STRUCT. 19 (9) (2007), 718-723.

7. Z.R. Lu, J.K. Liu, M. Huang, W.H. Xu, Identification of local damages in coupled beam systems from measured dynamic responses, J SOUND VIB. 326 (1-2) (2009)177189.

8. P. Cawley, R.D. Adams, The location of defects in structures from measurements of natural frequencies, $J$ STRAIN ANAL, 14 (2) (1979) 49-57.

9. M.A.B Abdo. M. Hori, A numerical study of structural damage detection using changes in the rotation of mode shapes, J SOUND VIB. 251 (2) (2002) 227-239.

10. A.K. Pandey, M. Biswas, M.M. Samman, Damage detection from changes in curvature mode shapes, $J$ SOUND VIB. 145 (2) (1991) 321-332.

11. Z.Y. Shi, S.S. Law, Structural damage localization from modal strain energy change, J SOUND VIB. 218 (5) (1998) 825-844.

12. Z. Zhang, A.E. Aktan, Application of modal flexibility and its derivatives in structural identification, RES NONDESTRUCT EVAL. 10 (1) (1998) 43-61.

13. M.S. Cao, P.Z. Qiao, Novel Laplacian scheme and multiresolution modal curvatures for structural damage identification, MECH SYST SIGNAL PR. 23 (4) (2009), 1223-1242.

14. M. Chandrashekhar, R. Ganguli, Structural Damage Detection Using Modal Curvature and Fuzzy Logic, STRUCT HEALTH MONIT. 8 (4) (2009) 267-282.

15. C.P. Ratcliffe, Damage detection using a modified laplacian operator on modal shape data, J SOUND VIB. 204 (3) (1997) 505-517.

16. M.K. Yoon, D. Heider, J.J.W Gillespie, C.P. Ratcliffe, R.M. Crane, Local damage detection with the global fitting method using modal shape data in notched beams, J NONDESTRUCT EVAL. 29 (1) (2009) 63-74.

17. J.J. Lee, J.W. Lee, J.H. Yi, C.B. Yun, H.Y. Jung, Neural networks-based damage detection for bridges considering errors in baseline finite element models, $J$ SOUND VIB. 280 (3-5) (2005) 555-578.

18. H. Guan, V.M. Karbhari, Improved damage detection method based on Element Modal Strain Damage Index using sparse measurement, J SOUND VIB. 309 (3-5) (2008) 465-494.

19. J.M. Ko, Z.G. Sun, Y.Q. Ni, Multi-stage identification scheme for detecting damage in cablestayed Kap Shui Mun Bridge, ENG STRUCT. 24(7) (2002) 857-868.
20. M. Sahin, R.A. Shenoi, Quantification and localisation of damage in beam-like structures by using artificial neural networks with experimental validation, ENG STRUCT. 25(14) (2003) 1785-1802.

21. W.T. Yeung, J.W. Smith, Damage detection in bridges using neural networks for pattern recognition of vibration signatures. ENG STRUCT. 27(5) (2005) 685-698.

22. N. Bakhary, H. Hao, A.J. Deeks, Damage detection using artificial neural network with consideration of uncertainties, ENG STRUCT. 29(11) (2007) 2806-2815.

23. M. Mehrjoo, N. Khaji, H. Moharrami, A. Bahreinineja, Damage detection of truss bridge joints using Artificial Neural Networks, Expert Syst Appl. 35(3) (2008) 1122 1131.

24. R. Gauguli, A fuzzy logic system for ground based structural health monitoring of a helicopter rotor using modal data, J INTEL MAT SYST STR. 12(6) (2001) 397407.

25. Zadeh, Fuzzy logic = computing with words, IEEE $\mathrm{T}$ FUZZY SYST. 4 (2) (1996) 103-111.

26. P.M. Pawar, R. Ganguli, Genetic fuzzy system for online structural health monitoring of composite helicopter rotor blades, MECH SYST SIGNAL PR. 21(5) (2007) 22122236.

27. P. Beena, R. Ganguli, Structural damage detection using fuzzy cognitive maps and Hebbian learning, APPL SOFT COMPUT, 11(1) (2011) 1014-1020.

28. M. Chandrashekhar, R. Ganguli, Uncertainty handling in structural damage detection using fuzzy Logic and probabilistic simulation, MECH SYST SIGNAL PR. 23(2) (2009) 384-404.

29. Z. Zhao, C. Chen, A fuzzy system for concrete bridge damage diagnosis, Comput Struct 80(7-8) (2002) 629-641.

30. M.M. Reda Taha, J. Lucero, Damage identification for structural health monitoring using fuzzy pattern recognition, ENG STRUCT. 27(12) (2005) 1774-1783.

31. R. W. Clough, J. Penzien, Dynamics of Structures, 3nd edn. (Computers \& Structures, Inc., Berkeley, 1995)

32. A. Dutta, S. Talukdar, Damage detection in bridges using accurate modal parameters, Finite Elem Anal Des. 40(3) (2004) 287-304.

33. W.T. Yeung, J.W.Smith, Damage detection in bridges using neural networks for pattern recognition of vibration signatures, ENG STRUCT. 27(5) (2005) 685-698.

34. T.J. Ross, Fuzzy Logic with Engineering Applications, (McGraw-Hill, 1995)

35. W.V. Leekwijck, E.E. Kerre, Defuzzification: criteria and classification, FUZZY SET SYST. 108 (2) (1999) 159-178

36. H.B. Liu, Y.B. Jiao, Y.C. Cheng, Y.F. Gong, Reduction of uncertainties for damage identification of bridge based on fuzzy nearness and modal data, J APPL MATH. doi:10.1155/2012/812932 